\title{
C-16
}

\section{RESULTADOS PRÁCTICOS DE LA APLICACIÓN DE ESTANDARES INDUSTRIALES A LA INTEROPERABILIDAD EN EL REGADÍO: PROYECTO MEGA}

\author{
González, $D .{ }^{1}$, Calero, $R .^{2}$, Navarro, $M .{ }^{3}$, Iglesias, $S^{4}{ }^{4}$, Martín, $D .{ }^{5}$, Alcarria $R^{6}{ }^{6}$, Robles, \\ $T^{7}{ }^{3}$ López $M^{8}{ }^{8}$
}

${ }^{1}$ Ing. Agrónomo, Empresa de Transformación Agraria S.A. (TRAGSA), C/ Conde de Peñalver, 84, 28006 Madrid, dgonzal5@tragsa.es

2 Ing. Agrónomo, Empresa de Transformación Agraria S.A. (TRAGSA), C/ Conde de Peñalver, 84, 28006 Madrid, rcalero@tragsa.es

3 Ing. Telecomunicaciones, Empresa de Transformación Agraria S.A. (TRAGSA), C/ Conde de Peñalver, 84, 28006 Madrid, mnc@tragsa.es

${ }^{4}$ Ing. Agrónomo, Empresa de Transformación Agraria S.A. (TRAGSA), C/ Conde de Peñalver, 84, 28006 Madrid, siglesia@tragsa.es

${ }^{5}$ Doctor Ciencias de la Computación, Dpto. Ingeniería de Sistemas Telemáticos de ETSI Telecomunicaciones, Av. Complutense 30, 28040 Madrid, diego.martin.de.andres@upm.es

${ }^{6}$ Doctor Ing. Telecomunicación, Dpto. de Ingeniería Topográfica y Cartografía de la ETSI en Topografía, Geodesia y Cartografía, Carretera de Valencia, km 7, 28031 Madrid, ramon.alcarria@upm.es

${ }^{7}$ Doctor Ing. Telecomunicaciones, Dpto. de Ingeniería de Sistemas Telemáticos de la ETSI Telecomunicaciones, Av. Complutense 30, 28040 Madrid, tomas.robles@upm.es

8 Doctor Ing. Agrónomo, Empresa de Transformación Agraria S.A. (TRAGSA), C/ Conde de Peñalver, 84, 28006 Madrid, mlopez@tragsa.es

\section{Resumen}

La falta de estandarización en los sistemas de telecontrol de regadío dificulta enormemente la gestión y el mantenimiento de los mismos, pero no menos importante es reconocer que dificulta la explotación de las propias infraestructuras modernizadas. El proyecto MEGA define los criterios para establecer interoperabilidad entre los diferentes sistemas de control y gestión que se emplean en el regadío. Dicha interoperabilidad se basa en el establecimiento de una nueva arquitectura de control a través de la cual se comuniquen los diferentes sistemas mediante un lenguaje de modelización desarrollado exprofeso. El desarrollo teórico de la modelización y el diseño de la nueva arquitectura tienen un largo recorrido y son conocidos por todo el sector. Se pretende presentar a continuación cómo se ha procedido a la validación empírica de esas bases teóricas estableciéndose una interoperabilidad real entre diferentes sistemas, participando en las pruebas diferentes agentes del sector del regadío. 


\begin{abstract}
The lack of standardization of telecontrol systems for irrigation complicates their management and maintenance as well as the exploitation of modernized infrastructures. The MEGA project defines the criteria for establishing interoperability between different control and management systems used in irrigation. Such interoperability is based on the establishment of a new control architecture enabling the communication of various systems by a modeling language developed for this purpose. The theoretical development of modeling and design of the new architecture have a long way and are known throughout the sector. In this work we present the procedure to empirical validation of these theoretical bases, establishing a real interoperability between different systems, participating different operators from the irrigation sector in the testing.
\end{abstract}

\title{
1. Introducción y objetivos
}

Los sistemas de telecontrol son la cara más visible del regadío modernizado. Gran parte de sus potencialidades teóricas no han podido ser explotadas por dos grandes carencias en su concepción:

- Son soluciones cerradas e integrales. Incluyen hardware y software, ambos desarrollados atendiendo a las capacidades del fabricante del sistema y no a las necesidades de los usuarios finales. Por otro lado, pretenden abarcar ámbitos ligados pero que responden a necesidades diferentes: el control y la gestión.

- No están sometidos a normalización, al no existir estándares que definan especificaciones funcionales mínimas ni mecanismos de integración entre componentes de diferente fabricante.

Estas dos situaciones plantean limitantes críticos que producen ineficiencias en la explotación de infraestructuras modernizadas ya que:

- Dificultan el mantenimiento. No permiten una renovación y sustitución progresiva de equipamientos averiados y/u obsoletos si no es por los mismos elementos.

- Establecen barreras de acceso. Se establece una relación de dependencia entre los explotadores de las infraestructuras modernizadas y los instaladores del sistema de telecontrol elegido. La introducción de mejoras y actualizaciones de funcionalidades queda fuertemente restringida.

- Inhiben la aparición de aplicaciones de gestión especializadas. El foco de la modernización ha estado, hasta la fecha en los sistemas de control, dejando en un segundo plano la necesidad de gestionar las infraestructuras en base a criterios de eficiencia energética e hidráulica. Es en este punto donde reside actualmente el interés fundamental de los gestores y explotadores. La gestión coordinada entre agua y energía, o la generación de índices operacionales, no es asumible en la actualidad. Multitud de datos recogidos por los sistemas de 
telecontrol tienen una gran relevancia para la toma de decisión, pero no existen mecanismos para explotar esta potencialidad.

- Como no existe una caracterización unívoca de formatos, contenidos y dimensiones de los datos recogidos por los sistemas, los gestores se encuentran con informaciones de origen, formato y constitución diferentes. Esta ausencia de normalización en los datos acentúa la ineficiencia en la gestión.

Para contribuir a la problemática descrita surge el proyecto MEGA (Modelo Estandarizado de Gestión del Agua), con el objetivo de definir y desarrollar los criterios para establecer interoperabilidad entre los diferentes sistemas de control y gestión que se emplean en el regadío. El proyecto se centra en facilitar a los usuarios de instalaciones modernizadas las herramientas adaptadas a sus necesidades, buscando el máximo consenso con el resto de agentes involucrados: empresas del sector, administraciones y otros organismos del sector de la agricultura y la gestión de agua.

\section{Materiales y métodos}

El proyecto MEGA ha permitido elaborar un documento técnico sobre interoperabilidad entre sistemas de telecontrol. Este documento además, ha sido presentado y aprobado en la reunión ISO/TC 23/SC 18 - Remote monitoring and control systems del comité de normalización de ISO para que sea la base para la normalización de estos sistemas en términos de interoperabilidad. La estrategia que se ha seguido para resolver los problemas detectados se fundamenta en la normalización de tres ejes: modelización de regadío, definición de una nueva arquitectura interoperable y desarrollo de las herramientas informáticas necesarias para la aplicación de los dos primeros ejes.

Modelización de los elementos propios del regadío: informaciones básicas y operacionales.

La modelización se ha abordado empleando experiencias de otros sectores productivos a nivel internacional. Esta línea permite avanzar en la definición de soluciones sin fijar especificaciones tecnológicas que restrinjan el presente y el futuro del telecontrol y la gestión de regadíos. Define con fines de control y gestión, la estandarización de las infraestructuras hidráulicas del regadío. Se basa en el estándar internacional EN61512 - Control de los procesos de fabricación por lotes, conocido en el sector industrial como S-88. La metodología empleada aporta además herramientas para mejorar el control y la gestión, destacando:

- clasificación para los sistemas de control según con sus especificaciones constructivas, facilitando la correcta especificación de acuerdo con las necesidades de los usuarios. Esta clasificación permitirá reducir la dispersión en cuanto a calidad y funcionalidades entre sistemas.

- generación de indicadores de gestión de consumo de agua y eficiencia energética, entre otros. Con esos indicadores, el gestor podrá evaluar los resultados del manejo y mantenimiento de las instalaciones, ayudando a su mejora y corrección. 


\section{Nueva arquitectura software de los sistemas actuales, estableciendo los cauces para el lenguaje fruto de la modelización.}

Para alcanzar el objetivo de la interoperabilidad entre diferentes sistemas que operan en el regadío, no sólo debe estandarizarse el lenguaje común que emplearán, sino la arquitectura a la que todos estos sistemas deben responder. La nueva arquitectura definida por MEGA incluye dos interfaces normalizados a través de los cuales se empleará el lenguaje fruto de la modelización.

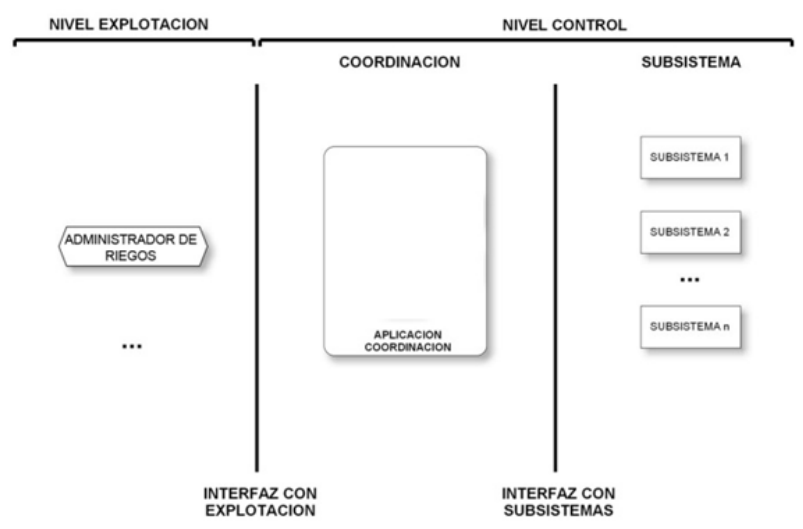

Figura 1: Arquitectura interoperable en las TICs del regadío

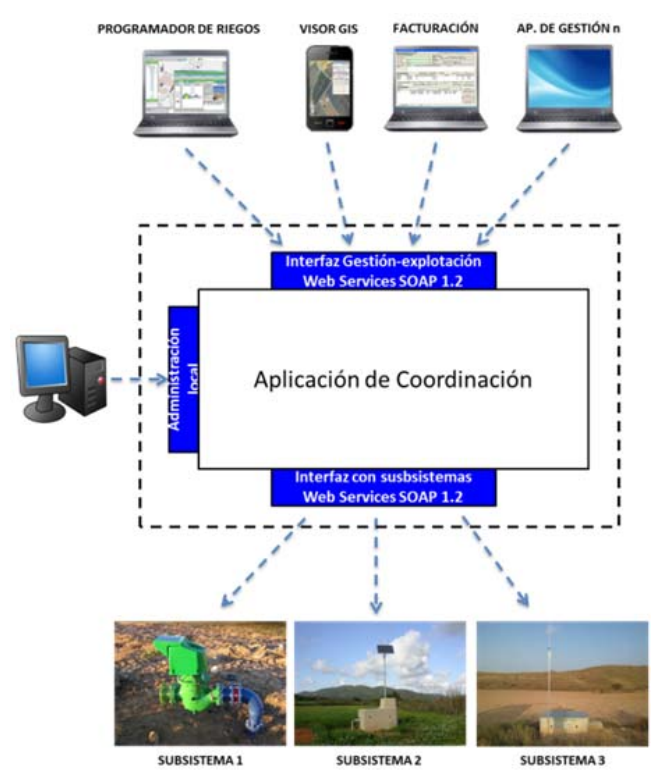

Figura 2: Aplicación de la arquitectura interoperable a las TICs del regadío

La nueva arquitectura pretende alcanzar una diferenciación clara entre dos mundos dependientes pero distintos:

- Nivel de gestión/explotación, orientado a la toma de decisiones.

- Nivel de control, dividido a su vez en dos subniveles, se orienta a la transmisión y adquisición de datos, así como a la ejecución de los procesos que se realizan en una entidad hidráulica. 
Esta nueva arquitectura será tendente a la especialización de los elementos que la componen.

Desarrollo de las herramientas necesarias para habilitar la nueva arquitectura que usa el lenguaje de modelización: la aplicación de coordinación.

La Universidad Politécnica de Madrid y Tragsa han colaborado en el desarrollo de la primera aplicación de coordinación con el fin de validar el lenguaje y la arquitectura definidos en el estándar, así como proporcionar los elementos comunes para el acoplamiento e interconexión de los sistemas de control y gestión del mercado. La aplicación de coordinación sustenta la interoperabilidad mediante la implementación de las siguientes funciones:

- Función 1. Mapeado/carga de entidades hidráulicas.

- Función 2. Monitorización de entidades hidráulicas.

- Función 3. Gestión de recetas de operación (programas de riego).

- Función 4. Envío de peticiones y recogida de respuestas.

- Función 5. Comunicación con los sistemas de telecontrol (subsistemas).

- Función 6. Comunicación con las aplicaciones de gestión.

- Función 7. Repositorio de datos.

Implementa dos interfaces de comunicación usando la tecnología de Servicios Web (o Web Services) para comunicarse con sistemas de telecontrol (interfaz con subsistemas) y con aplicaciones de gestión (interfaz con explotación). Recibe peticiones a través del protocolo SOAP (Simple Object Access Protocol) de la capa de gestión y realiza peticiones SOAP a la capa de subsistemas.

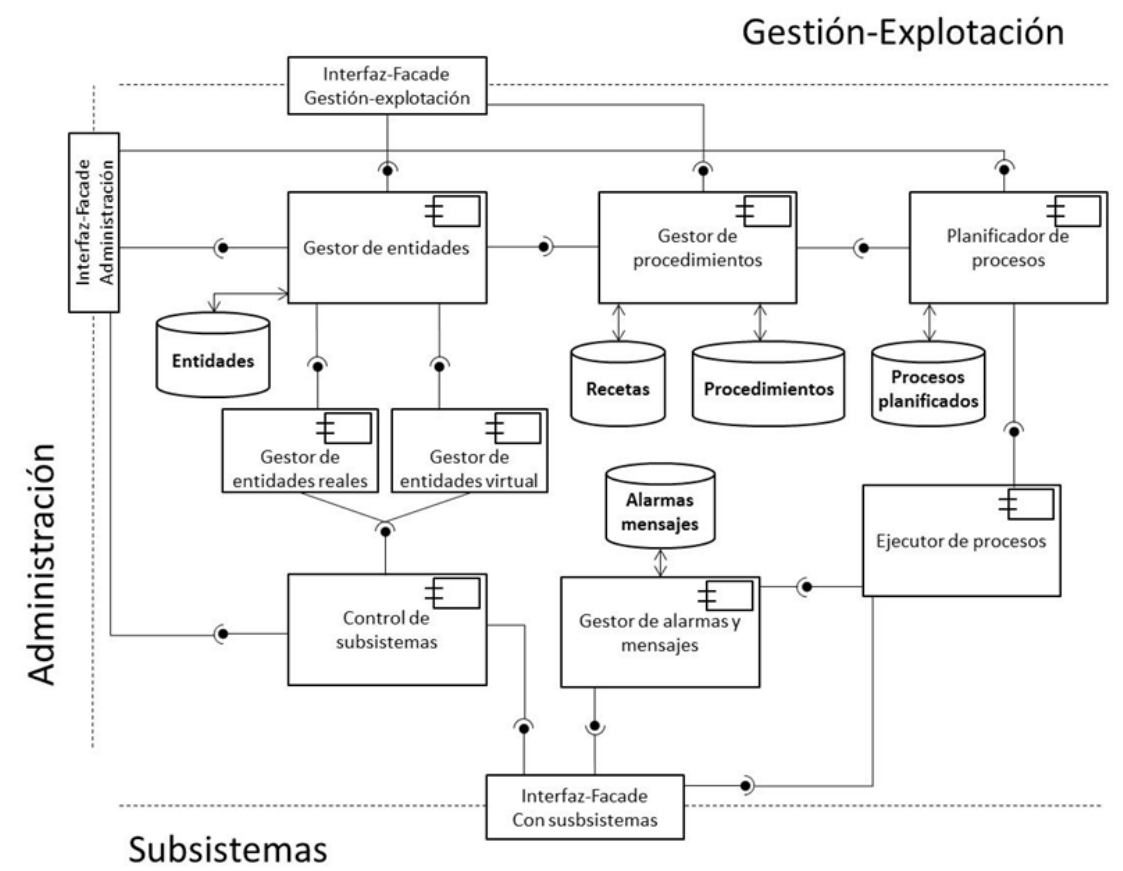

Figura 3: Diagrama de módulos que conforman una aplicación de coordinación 


\section{Resultados y discusión}

La adaptación de los sistemas de gestión y telecontrol al estándar de interoperabilidad definido, se ha verificado mediante la aplicación de una batería de ensayos sobre un conjunto de sistemas que han participado como pioneros. Las pruebas realizadas en el banco de ensayos de Aula Dei, centro de investigación que forma parte del CSIC, se basan en la especificación de un protocolo de ensayos que será presentado en ISO para avanzar en el estándar de interoperabilidad a nivel internacional.

Las pruebas se han iniciado en 2016 y continúan actualmente en desarrollo, continuando los participantes los desarrollos necesarios para adaptarse completamente a la especificación de MEGA. Actualmente la adaptación es parcial pero ilustrativa de los objetivos y metas esperables a corto plazo. Los participantes en esta fase de pruebas inicial son un núcleo de empresas con un claro interés estratégico en liderar la adaptación de los sistemas tradicionales de control y gestión del regadío:

- Tragsa, Universidad Politécnica de Madrid y EEAD-CSIC como organizadores e impulsores del proyecto MEGA y supervisores en las pruebas de validación.

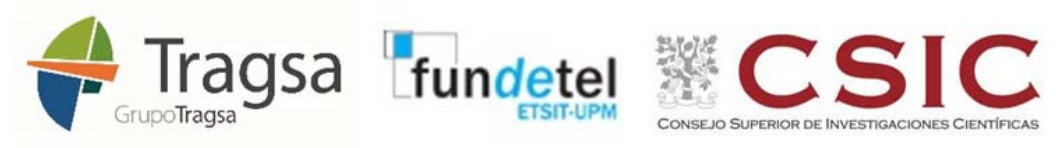

- $\quad$ ICR, Regaber e Isastur como empresas fabricantes de sistemas de telecontrol.
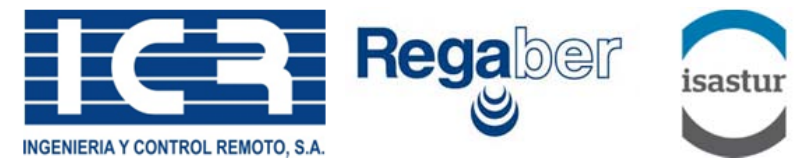

- Batchline Control y Serina como empresas desarrolladoras de aplicaciones de gestión (módulos de facturación y programación de riegos).
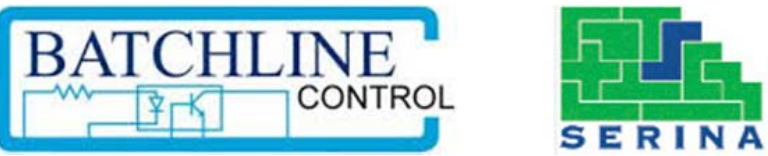

Figura 4: Participantes en las pruebas de validación del prototipo de interoperabilidad

\section{Entorno de pruebas}

Las pruebas necesarias para verificar la interoperabilidad se han realizado en un banco de ensayos diseñado para tal fin y ubicado en, estación experimental de Aula Dei, adscrita al Centro de Investigaciones Científicas (CSIC). El banco de ensayos replica un sistema completo, desde la acometida hasta el hidrante, con el fin de poder extender la interoperabilidad a cualquier infraestructura típica del regadío. 




Figura 5: Banco de ensayos de interoperabilidad (Aula Dei)

La instalación dispone, entre otros elementos, de 40 hidrantes denominados hidrantes (HYS) simples en la modelización, con un hidrómetro de cabecera. El esquema hidráulico característico de un hidrante simple (replicado en el banco de ensayos) es el siguiente:
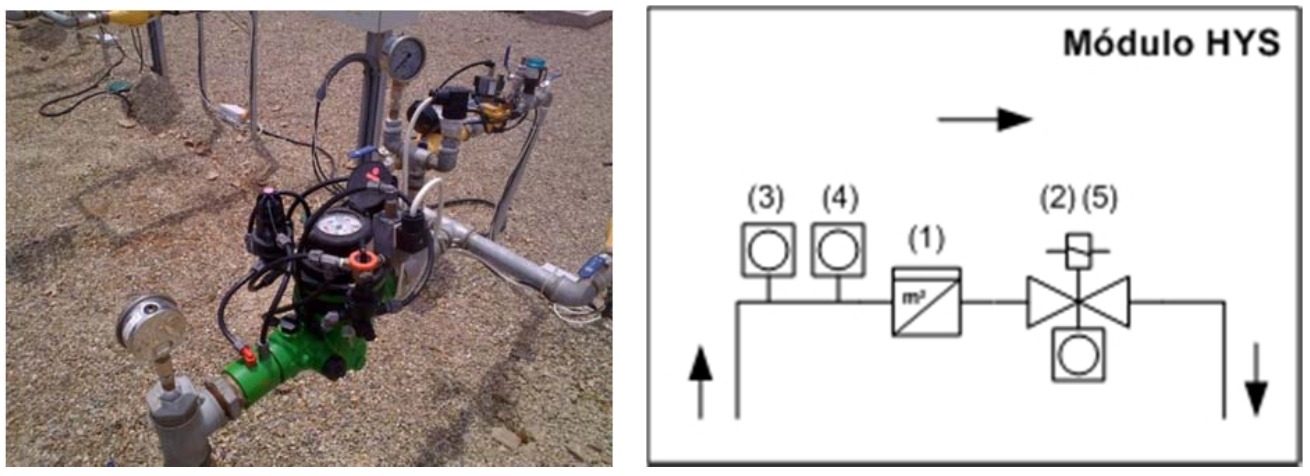

Figuras 6 y 7 - Fotografía de un módulo de pruebas de hidrante y diagrama de componentes de un hidrante simple (HYS)

Los componentes del módulo identificados en el diagrama son:

(1) Totalizador de volumen

(2) Elemento de corte controlado por subsistema

(3) Medidor de presión

(4) Detector de paso de agua

(5) Detector de posición de elemento de corte

A su vez, el banco dispone de la infraestructura informática necesaria para comprobar y validar la arquitectura propuesta para los sistemas de control y gestión. El control de los diferentes elementos incluidos en el banco se basa en la instalación del software necesario en máquinas virtuales. Cada subsistema, aplicación de gestión y/o 
aplicación de coordinación se instala en una máquina virtual independiente a la que sólo tienen acceso los organizadores de los ensayos y los propietarios de la misma. Todos los elementos hidráulicos que componen el banco de ensayos disponen de conexión directa con la red informática, disponiendo a su vez de suministro energético para los sistemas de control que se desee testar.

Se establece la siguiente metodología para la ejecución de los ensayos sobre el sistema o aplicación en pruebas, de acuerdo con las exigencias fijadas por el estándar para la explotación y el control de cada tipo de entidad hidráulica. El fabricante 0 desarrollador deberá informar de cuáles son las entidades susceptibles de control o explotación por su producto.

Todo sistema o aplicación susceptible de ser compatible con el presente estándar se somete al procedimiento de ensayo, divido en la siguiente secuencia de fases:

- Fase 1. Pruebas informáticas de verificación de interfaz. Se verifica que los mensajes (tanto peticiones como respuestas) que intercambian los componentes de la arquitectura que se están probando (aplicaciones de gestión, sistemas de telecontrol y aplicación de coordinación) son acordes con el estándar de comunicaciones definido para esos componentes.

- Fase 2. Ensayos de campo para verificación de funcionalidades. Una vez superada la fase anterior, se verifica que los sistemas interoperables, actuando de forma conjunta, ejecutan correctamente las funcionalidades que se les asocian.

Las pruebas realizadas están orientadas a las funciones básicas que deben resolver los sistemas de gestión y de telecontrol de regadíos, siendo que el estándar de interoperabilidad incluye otras funcionalidades que serán testadas en el futuro. Las pruebas realizadas se han orientado a dos funciones básicas que se atribuyen a estos sistemas:

- La facturación;

- y la programación de riegos.

Para validar el correcto desarrollo de estas funcionalidades de acuerdo con los criterios establecidos por el estándar de MEGA se ha realizado la siguiente batería de pruebas básicas:

- Prueba 1. Facturación sin discriminación horaria. Obtener la facturación correspondiente a dos o más hidrantes, controlados por diferentes subsistemas, obteniendo los datos necesarios para que un único software los incorpore con el fin de realizar la facturación.

- Prueba 2. Ejecución de riego programado finalizado por tiempo. Programar uno o más riegos con finalización por tiempo, al menos en tres hidrantes controlados por diferentes subsistemas, lanzando la petición a los mismos desde un único software de programación de riegos. Condición de fin: tiempo máximo.

- Prueba 3. Ejecución de riego programado finalizado por volumen. Programar uno o más riegos con finalización por volumen, al menos en tres hidrantes 
controlados por diferentes subsistemas, lanzando una petición a los mismos desde un único software de programación de riegos. Condiciones de fin: tiempo máximo y volumen máximo.

- Prueba 4. Ejecución de riego programado finalizado por petición directa. Programar uno o más riegos con finalización por tiempo, al menos en tres hidrantes controlados por diferentes subsistemas, lanzando la petición de paro desde un único software de programación de riegos. Una vez se haya iniciado el riego, se enviará la orden de paro.

Estas pruebas se han realizado de forma pautada, atendiendo al protocolo de ensayos de MEGA que, junto con los anexos de implementación, es otro de los documentos presentados en los comités de normalización.

\section{Conclusiones y recomendaciones}

Con la batería de pruebas definidas, se consigue cerrar una primera fase en la interoperabilidad, en la que se ha tratado de validar la línea de trabajo mantenida a lo largo del desarrollo teórico. Se demuestra empíricamente que sistemas de telecontrol de diferentes fabricantes pueden explotarse desde aplicaciones pensadas para la gestión de las infraestructuras modernizadas, también diseñadas y desarrolladas por terceros.

Las potencialidades de MEGA se verán traducidas, en los próximos tiempos en:

- Valorización de las herramientas de gestión, ahora infrautilizadas y con un amplio margen de implantación para la mejora de la explotación y el mantenimiento en las áreas regables. En línea con lo anterior, se abre el abanico al desarrollo de nuevas herramientas de gestión que cubran aspectos que actualmente son deficitarios al tratarse de condicionantes nuevos que no existían en el momento en el que se diseñaron las soluciones actuales (gestión coordinada y eficiente de agua y energía).

- Desligamiento entre usuarios y fabricantes de sistemas de telecontrol, permitiendo la elección de la mejor solución en cada momento, fomentándose la competitividad y mejora de los productos ofertados por el sector. El desligamiento permite abrir un mercado de prestación de servicios de explotación y mantenimiento, que también contribuirá a reducir el abandono de los sistemas y la potenciación de las informaciones que de ellos se pueden obtener.

Entre 2000 y 2014 , se ha modernizado casi el $40 \%$ de la superficie de regadío en España, alcanzando los 1,5 millones de hectáreas modernizadas sobre los 3,6 millones de hectáreas totales en $2014^{1}$, repartidas entre 7200 Comunidades de Regantes. De acuerdo con datos de la Junta de Castilla y León, el coste de explotación se ubica entorno a los 700 euros por hectárea ${ }^{2}$. No debe obviarse que la

1 Aumenta la superficie regada en España pero se mantiene una tendencia a la baja en el volumen de agua de riego utilizado en el sector agrario. Nota de prensa MAGRAMA. 18 de marzo de 2015.

2 Coste del riego según tipo de infraestructura. Portal www.inforiego.org. Instituto Tecnológico Agrário de Castilla y Leon (ITACyL), Datos campaña agrícola 2012-2013. 
tendencia de ese valor es a incrementarse (coste de energía, coste de uso del recurso agua por aplicación de la DM del Agua).

A nivel Comunidad de Regantes, y desde el punto de vista técnico, la implementación de elementos estandarizados e interoperables, facilitará las tareas de explotación y mantenimiento consiguiendo mayores eficiencias en el nexo agua-energía. Al ahorro conseguido por la mejora de la eficiencia hidro-energética, hay que sumar los que se conseguirán al tener mejores precios en la reposición de elementos de control al dejar de ser las zonas regables "esclavas" de una marca determinada y poder seleccionar las compras dentro de un marco de competencia comercial. Es por ello que la interoperabilidad contribuye de forma significativa al incremento de los márgenes económicos y a la viabilidad económica de las modernizaciones realizadas en los últimos 15 años.

\section{Bibliografía}

- $\quad$ EN 61512: Batch control - Part 2: Data structures and guidelines for languages. EN 61512-2:2002.

- EN 62264: Enterprise-control system integration - Part 1: Models and terminology. EN 62264-1:2013.

- EU Water Framework Directive (Directive 2000/60/EC), 23 October 2000.

- $\quad$ ISO 9646 Conformance Testing Methodology and Framework. 